\title{
Daptomycin-Based 3 Week Treatment Scheme for the First Cellulitis Episode: Low Recurrence Rate Indicative of Decreased Recurrence Propensity?
}

\author{
Ioannis D. Bassukas (D) - Ioannis Chaniotakis · Georgios Gaitanis
}

To view enhanced content go to www.dermtherapy-open.com Received: November 24, 2015 / Published online: February 2, 2016

(c) The Author(s) 2016. This article is published with open access at Springerlink.com

\begin{abstract}
Introduction: Cellulitis recurrences are frequent, difficult to manage, and contribute considerably to overall disease burden. This retrospective cases series reports recurrence rates of first community-acquired lower limb disease episode initially treated with daptomycin.
\end{abstract}

Methods: Treatment consisted of a 21-day two substances sequential scheme: intravenous daptomycin $\quad(4 \mathrm{mg} / \mathrm{kg} /$ day $) \quad$ during hospitalization and roxithromycin $(2 \times 150 \mathrm{mg}$ per os) afterwards. Primary study end-point was the rate of recurrences during follow-up; recurrence rates and their binomial 95\% confidence intervals (CI) were calculated with SPSS 22.0. Recurrences were identified by (1)

Electronic supplementary material The online version of this article (doi:10.1007/s13555-016-0095-8) contains supplementary material, which is available to authorized users.

I. D. Bassukas $(\bowtie) \cdot$ I. Chaniotakis · G. Gaitanis Department of Skin and Venereal Diseases, Faculty of Medicine, School of Health Sciences, University of Ioannina and University Hospital of Ioannina, Ioannina, Greece

e-mail: ibassuka@cc.uoi.gr review of hospital records and (2) telephone questionnaires.

Results: Twenty-seven patients hospitalized for first lower limb cellulitis episodes were started on daptomycin and 26 patients completed the above three-week sequential treatment scheme. During follow-up (range 37-85 months) one recurrence occurred among the 26 patients who completed the treatment: $96.2 \%$ of these patients remained relapse free at the end of follow-up (CI 79.5-99.9\%). Considering also treatment failure, the overall effectiveness of this combination treatment was $92.6 \%$ recurrence free after more than 3 years follow-up (25/27 patients; CI 75.5-99.0\%). This study is limited by retrospective planning and the restricted number of included patients. Conclusion: The choice of the treatment for the initial lower limb cellulitis episode may impact the risk of disease recurrences. Future studies should explore interventions for the first cellulitis episode as a means to modify the risk of the recurring course of this disease.

Keywords: Cellulitis; Daptomycin; Erysipelas; Leg; Lower limb; Recurrence; Roxithromycin 


\section{INTRODUCTION}

Cellulitis is a common bacterial skin infection that involves the lower extremities in $>70 \%$ of cases $[1,2]$. Recurrences are frequent affecting $15-40 \%$ of patients in the first 3 years and constitute a significant risk factor for further disease episodes [1, 3]. Common causative agents comprise Gram-positive aerobes, particularly Streptococci (groups A, B, C and G) and Staphylococcus aureus [2, 4]. Widely used recommendations for initial empirical treatment include short schemes (5-10 days) with beta-lactam antimicrobials [2].

This retrospective case series reports a reasonably low recurrence rate in patients treated with a 3-week sequential daptomycin-roxithromycin scheme for the initial lower limb cellulitis episode.

\section{METHODS}

Adult patients hospitalized in the Dermatology Clinic of the Ioannina University Hospital between January 2008 and December 2011 for the first episode of community-acquired lower limb cellulitis were treated sequentially with intravenous daptomycin (4 mg/kg/day; Cubicin $^{\circledR}$, Novartis Pharmaceuticals, UK, Ltd) during hospitalization and roxithromycin per os $\left(2 \times 150 \mathrm{mg}\right.$ daily; Rulid ${ }^{\circledR}$, Sanofi-Aventis A.E.B.E., Greece) for a total of three weeks. Cases requiring hospitalization (for treatment strategy of patients with cellulitis in this hospital see also [3]) were defined as patients with clinical findings of cellulitis (unilateral, relatively demarcated area of expanding skin inflammation, with malaise, fever or chills) and an abnormal result in at least one of the following tests: total white blood cell (WBC) count (normal range: $4500-10,500$ cells $/ \mu \mathrm{L}$ ), serum C-reactive protein (CRP) level (normal range $<6 \mathrm{mg} / \mathrm{L}$ ) or erythrocyte sedimentation rate (ESR; normal range $<20 \mathrm{~mm} / \mathrm{h}$ ). Only patients who completed treatment were considered in the recurrences analysis. Primary study end-point was the rate of recurrences during follow-up after treatment, that is, cellulitis in the same lower limb diagnosed more than 1 month after treatment of the first disease episode. Recurrences were identified by (1) hospital records (archived patients' files) of cellulitis admissions and (2) telephone questionnaires conducted in February 2012 and February 2015. Recurrence rates and their binomial 95\% confidence intervals (CIs) were calculated with SPSS 22.0 software package (SPSS Inc., Chicago, IL, USA). Moreover, employing the mean individualized 2 years follow-up recurrence probabilities of lower leg cellulitis according to the prognostic model of McNamara et al. [5] an anticipated recurrence rate was calculated for the present case series. For this study University Hospital of Ioannina Ethics and Clinical Trials Review Committee permission was granted according to the Declaration of Helsinki Principles.

\section{RESULTS}

Twenty-seven patients were started on intravenous daptomycin and 26 patients (15 male, 11 female; median age 68 years, range 38-89 years) who completed treatment were followed up for recurrences. Depending on the individual disease course the patients received daptomycin for 7-14 days. One patient with inadequate clinical cellulitis control at $48 \mathrm{~h}$ after treatment initiation was excluded from follow-up: treatment failure rate of daptomycin $3.7 \%$. In the case of this single non-responder cure was achieved by adding intravenous 
ceftriaxone ( 2 g/day; Rocephin ${ }^{\circledR}$, Roche Hellas A.E.) to the treatment scheme. After 37-85 months follow-up one recurrence occurred, that is, $96.2 \%$ of the patients remained relapse-free at the end of follow-up (95\% CI 79.5-99.9\%). The recurrence occurred in a 70-year-old female patient with a left tibia cellulitis and history of breast cancer (see Table S1 in the supplementary material for a compilation of core cohorts' demographic and disease parameters). It is worth noting that the predicted recurrence free rate at 2 years follow-up according to McNamara's recurrence score for lower leg cellulitis $(73.5 \%)$ is outside the $95 \%$ confidence range of the recurrence rate observed in the present case series. However, instead of the 2-year period of the above model we presently considered follow-up periods $>3$ years. Finally, considering overall effectiveness of the treatment, 25/27 patients initially treated with daptomycin for the first lower leg cellulitis episode (92.6\%; 95\% CI 75.5-99.0\%) remain recurrence free after more than 3 years follow-up.

\section{DISCUSSION}

With the present three-week treatment combination, in addition to a satisfactory $(>95 \%)$ cure rate, we observed an optimistic low disease recurrence rate after more than 3 years follow-up. The present observation further supports our recent finding that the treatment of the first cellulitis episode with a 3 week clindamycin scheme may reduce the risk of subsequent recurrences [3]. Compared to conventional treatment selections cure rate of cellulitis with daptomycin are not inferior and due to once daily bolus administration, this agent could be also suitable for outpatient parental antibiotic therapy $[6,7]$.

The pathogenesis of recurrent cellulitis episodes is still poorly understood $[1,2]$. Recurrent episodes of cellulitis generate functional residua in the affected skin area that predispose to subsequent disease episodes, thus creating a vicious cycle. To date the concept of modifying cellulitis recurrence risk by the selection of different treatment schemes for the first disease episode has not been adequately addressed. However, the treatment choice for the first cellulitis episode may confer protection against the risk of future relapses by different mechanisms: (1) Targeting bacterial protein synthesis by the antibacterial scheme could inhibit toxin synthesis and inflammatory mediators production during infection and thus protect tissues from excess damage (e.g., lymphedema perpetuation) [3, 4]; (2) prolonged treatment duration ( $>2$ weeks) may effectively eradicate latent infection foci in the relatively immunocompromised compartment of the tibial skin [8, 9]; (3) It is further conceivable that the pathogenetic potential of the infecting bacterial strain, as denoted by the species and subspecies status, could variably predispose to subsequent relapses [10]. Daptomycin and roxithromycin target a broader species spectrum than traditional beta-lactam antibacterials.

This may be the first report of a long-term follow-up for recurrences in patients who were started on daptomycin for the first cellulitis episode. This study is limited by retrospective planning and the restricted number of included patients. Future studies should explore interventions for the first cellulitis episode as a means to modify the risk of the frequently detrimental recurring course of this disease. 


\section{ACKNOWLEDGMENTS}

No funding or sponsorship was received for this study. The publication of this article (processing charges) was supported by the University of Ioannina Research Committee Account No 22195. All named authors meet the International Committee of Medical Journal Editors (ICMJE) criteria for authorship for this manuscript, take responsibility for the integrity of the work as a whole, and have given final approval to the version to be published.

Disclosures. Ioannis D. Bassukas, Ioannis Chaniotakis, and Georgios Gaitanis have nothing to disclose.

Compliance with Ethics Guidelines. All procedures followed were in accordance with the ethical standards of the responsible committee on human experimentation (institutional and national) and with the Helsinki Declaration of 1964, as revised in 2013. This article is a retrospective observational study based on previously conducted treatments.

Open Access. This article is distributed under the terms of the Creative Commons Attribution-NonCommercial 4.0 International License (http://creativecommons.org/licenses/ by-nc/4.0/), which permits any noncommercial use, distribution, and reproduction in any medium, provided you give appropriate credit to the original author(s) and the source, provide a link to the Creative Commons license, and indicate if changes were made.

\section{REFERENCES}

1. Hirschmann JV, Raugi GJ. Lower limb cellulitis and its mimics. Part I. Lower limb cellulitis. J Am Acad Dermatol 2012;67:163.e1-12.

2. Stevens DL, Bisno AL, Chambers HF, et al. Practice guidelines for the diagnosis and management of skin and soft tissue infections: 2014 update by the Infectious Diseases Society of America. Clin Infect Dis. 2014;59:e10-52.

3. Chaniotakis I, Gaitanis G, Skandalis K, Alexis I, Bassukas ID. Reduced recurrence rate after a three week clindamycin for the initial lower limb cellulitis. J J Expt Derm Res. 2015;1:009.

4. Horseman M, Bowman JD. Is community-acquired methicillin-resistant Staphylococcus aureus coverage needed for cellulitis? Infect Dis Ther. 2013;2:175-85.

5. McNamara DR, Tleyjeh IM, Berbari EF, et al. A predictive model of recurrent lower extremity cellulitis in a population-based cohort. Arch Intern Med. 2007;167:709-15.

6. Arbeit RD, Maki D, Tally FP, et al. The safety and efficacy of daptomycin for the treatment of complicated skin and skin-structure infections. Clin Infect Dis. 2004;38:1673-81.

7. Seaton RA, Barr DA. Outpatient parental antibiotic therapy: principles and practice. Eur J Intern Med. 2013;24:617-23.

8. Cox NH. Oedema as a risk factor for multiple episodes of cellulitis/erysipelas of the lower leg: a series with community follow-up. Br J Dermatol. 2006;155:947-50.

9. Carlson JA. Lymphedema and subclinical lymphostasis (microlymphedema) facilitate cutaneous infection, inflammatory dermatoses, and neoplasia: a locus minoris resistentiae. Clin Dermatol. 2014;32:599-615.

10. Komatsu Y, Okazaki A, Hirahara K, Araki K, Shiohara T. Differences in clinical features and outcomes between group A and group G Streptococcus-induced cellulitis. Dermatology. 2015;230:244-9. 